\title{
Aseptic Meningitis with Craniopharyngioma Resection: Consideration after Endoscopic Surgery
}

\author{
Jenny X. Chen ${ }^{1}$ Blake C. Alkire ${ }^{1,2}$ Allen C. Lam ${ }^{1,2}$ William T. Curry ${ }^{3 *}$ Eric H. Holbrook ${ }^{1,2 *}$
}

${ }^{1}$ Massachusetts Eye and Ear Infirmary, Boston, Massachusetts, United States

2 Department of Otolaryngology, Harvard Medical School, Boston, Massachusetts, United States

${ }^{3}$ Department of Surgery (Neurosurgery), Harvard Medical School, Boston, Massachusetts, United States

\begin{abstract}
Address for correspondence Jenny X. Chen, MD, Massachusetts Eye and Ear Infirmary, 243 Charles Street Boston, MA, 02114, United States (e-mail: jenny_chen@meei.harvard.edu).
\end{abstract}

J Neurol Surg Rep 2016;77:e151-e155.

\begin{abstract}
Keywords

- skull base surgery

- complications

- cerebral vasospasm

- CSF lactate

Objectives While bacterial meningitis is a concerning complication after endoscopic skull base surgery, the diagnosis can be made without consideration for aseptic meningitis. This article aims to (1) present a patient with recurrent craniopharyngioma and multiple postoperative episodes of aseptic meningitis and (2) discuss the diagnosis and management of aseptic meningitis.

Design Case report and literature review.

Results A 65-year-old female patient with a symptomatic craniopharyngioma underwent transsphenoidal resection. She returned postoperatively with symptoms concerning for cerebrospinal fluid (CSF) leak and bacterial meningitis. Lumbar puncture demonstrated mildly elevated leukocytes with normal glucose levels. Cultures were sterile and she was discharged on antibiotics. She returned 18 days postoperatively with altered mental status and fever. Again, negative CSF cultures suggested aseptic meningitis. Radiological and intraoperative findings were now concerning for widespread cerebrovascular vasospasm due to leaked craniopharyngioma fluids. In the following months, her craniopharyngioma recurred and required multiple surgical resections. Days after her last operation, she returned with mental status changes and a sterile CSF culture. She was diagnosed with recurrent aseptic meningitis and antibiotics were discontinued. The patient experienced near complete resolution of symptoms. Conclusions Consideration of aseptic meningitis following craniopharyngioma resection is critical to avoid unnecessary surgical re-exploration and prolonged courses of antibiotics.
\end{abstract}

\section{Introduction}

Bacterial meningitis is a rare but potentially life-threatening complication of endoscopic skull base surgery, occurring in fewer than $5 \%$ of all cases. ${ }^{1}$ Symptoms of meningismus can indicate bacterial infection, with additional concern for a These authors contributed equally to this work and are cosenior
authors.

received

January 19, 2016

accepted

DOI http://dx.doi.org/

August 29, 2016 failed repair and persistent cerebrospinal fluid (CSF) leak. However, aseptic or chemical meningitis can present with similar symptoms, including fever, headache, nuchal rigidity, and photophobia, and represents 50 to $75 \%$ of all postsurgical cases of meningitis. ${ }^{1,2}$ Aseptic meningitis occurs secondary to chemical irritation of the meninges and is most commonly triggered by surgical manipulation or blood. ${ }^{3-5}$ Just as for other cystic lesions, intracranial rupture of craniopharyngiomas has also been proposed as an independent cause of
License terms

Stuttgart - New York

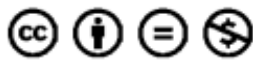


aseptic meningitis as cholesterol crystals released from the cyst cavity can induce a chemically mediated inflammatory response severe enough to trigger cerebrovascular vasospasm. ${ }^{6}$ While aseptic meningitis can be difficult to diagnose, it is imperative that it be considered in patients after skull base surgery to avoid unnecessary long-term antibiotic treatment for infectious meningitis or surgical re-exploration for suspected CSF leak. We present a case of a patient with multiple episodes of aseptic meningitis culminating in cerebral infarction from vasospasm after endoscopic resection of a craniopharyngioma. In addition, an algorithm for differentiating aseptic from bacterial meningitis is provided along with an evidence-based discussion of the diagnosis and treatment of patients with this rare complication.

\section{Case Study}

A 65-year-old female patient presented with a 2-month history of progressive diplopia and fatigue. She was found to have a bitemporal hemianopsia. Magnetic resonance imaging (MRI) demonstrated a $2.5 \times 2.4 \times 2.1 \mathrm{~cm}$, mixed cystic and solid mass suggestive of a craniopharyngioma with mass effect on the optic chiasm and tracts ( - Fig. 1). Given the progression of her symptoms, the patient underwent an image-guided endoscopic transsphenoidal, transsellar decompression of the suprasellar mass. A CSF leak was encountered intraoperatively, and the skull base defect was repaired with an abdominal fat graft followed by a sheet of the acellular dermis and middle turbinate bone graft in an underlay fashion. A pedicled septal mucosal graft was then used to cover the repair. The pathology

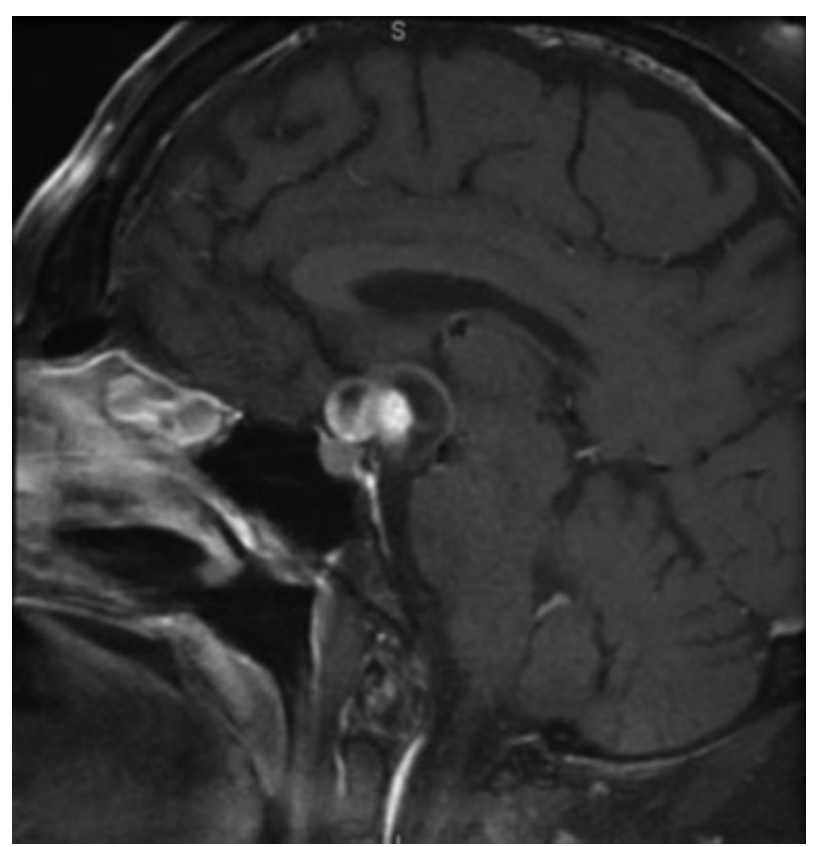

Fig. 1 T1-weighted sagittal MRI with gadolinium enhancement. A mixed cystic and solid mass is seen superior and posterior to the sella with the overall dimensions measuring $2.5 \mathrm{~cm} \mathrm{AP} \times 2.4 \mathrm{~cm}$

TV $\times 2.1 \mathrm{~cm} \mathrm{SI}$. The mass resides entirely in the suprasellar region. The mass results in superior displacement of the optic chiasm. AP, anteriorposterior; MRI, magnetic resonance imaging; SI, superior-inferior; TV, transversal. results returned as papillary-type craniopharyngioma. Postoperatively, she had improved vision with no evidence of CSF leak, and was discharged to a rehabilitation facility in good condition on postoperative day 6 .

After 2 days after discharge, she presented to the emergency department with fever to $101.7^{\circ} \mathrm{F}$ and fatigue. She denied meningismus or active nasal drainage. Her neurological examination was nonfocal. Her serum white blood cell count was mildly elevated at 13,000 cells $/ \mu \mathrm{L}$, although this was difficult to interpret in the setting of postoperative steroid use and peripheral leukocytosis. An MRI demonstrated a focus of restricted diffusion around the operative site with no leptomeningeal enhancement. A lumbar puncture was notable for the presence of 322 total nucleated cells $/ \mu \mathrm{L}$ with a neutrophil predominance, normal glucose, and elevated total protein levels. The infectious disease service was consulted, and the patient was started on vancomycin and ceftriaxone for presumed bacterial meningitis. She improved clinically during her hospitalization and quickly defervesce. Her CSF cultures returned sterile. However, with lingering suspicion for a bacterial infection, she was discharged with plans for 3 weeks of intravenous antibiotics, followed by an additional week of oral antibiotics.

After 18 days of her original transsphenoidal surgery, the patient returned with right facial paresis, right-sided weakness, and headache. CSF analysis again showed mild leukocytosis at 55 cells $/ \mu \mathrm{L}$, normal glucose, and elevated total protein. A repeat MRI demonstrated regrowth of the supracellar cyst, as well as multiple foci suspicious for acute/subacute infarction in the left anteromedial temporal lobe, putamen, corona radiata, and cerebral peduncle. The magnetic resonance angiography demonstrated multifocal stenosis in the proximal branches of the left anterior communicating artery and the left middle cerebral artery concerning for emboli ( - Fig. 2). In addition, the patient had a rim-enhancing lesion in the left temporal lobe initially concerning for an abscess for which she was taken to the operating room for a left craniotomy and washout. Intraoperatively, a yellow sludge-like material with the consistency of thick glue was found in the cisterns, suspected to be fluid from a cystic craniopharyngioma. CSF fluid and surgical cultures taken from near the left temporal tip returned sterile, although infectious diseases consultants recommended that she initiate a 4-week course of meropenem and vancomycin. On follow-up MRIs, the left temporal lesion became confirmed as an area of subacute infarction rather than an abscess consistent with the intraoperative findings.

Several weeks after discharge, the patient developed worsening of her vision once again. Another MRI demonstrated reaccumulation of the cystic component in addition to the known residual solid component of her craniopharyngioma with compression on the optic chiasm (-Fig. 3). She underwent a right pterional craniotomy for decompression and subtotal resection of the mass. The craniopharyngioma subsequently regrew over the next month and she was again taken to the operating room for a right frontotemporal orbitozygomatic craniotomy for resection. She recovered and went to a rehabilitation facility in good condition after both the operations. 


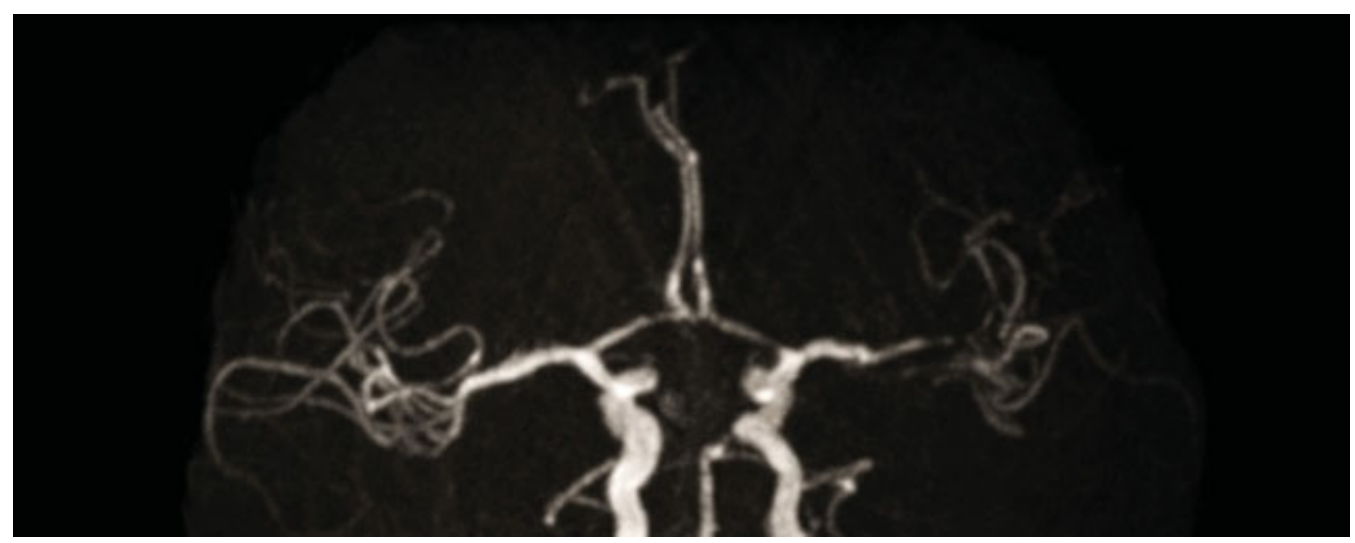

Fig. 2 MRA of the head on postoperative day 19 showing multifocal stenosis in the proximal A1 segment of the left anterior cerebral artery, distal M1/proximal M2 segments of the left middle cerebral artery, concerning for cerebral vasospasm versus septic emboli. MRA, magnetic resonance angiography.

Days after discharge from her last craniotomy, the patient returned to the emergency department febrile to $103^{\circ} \mathrm{F}$, hypotensive, and with altered mental status. A repeat MRI did not find evidence of an intracranial abscess. A lumbar puncture again demonstrated mild leukocytosis with left shift, elevated glucose, and elevated total protein. She was started on linezolid, meropenem, and micafungin. Cultures again returned sterile. At this time, she was diagnosed with recurrent episodes of severe aseptic meningitis. Antibiotics were discontinued at the recommendation of the infectious disease service. The patient improved clinically without negative sequelae after discontinuation of antibiotics. She eventually underwent radiation treatment for her recurrent craniopharyngioma. Her vision improved with only a residual

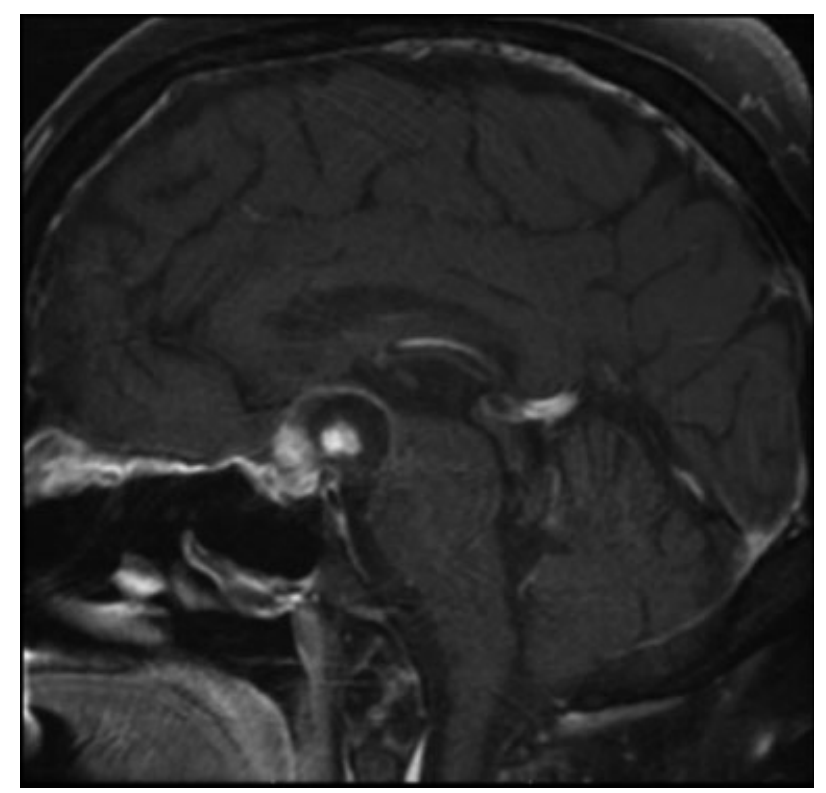

Fig. 3 Postoperative T1-weighted sagittal MRI. In the suprasellar cistern just superior to the dorsum sella, there is enhancing nodular tissue consistent with known residual solid mass, along with a new peripherally enhancing cystic mass measuring $2.5 \mathrm{~cm} \mathrm{AP} \times 2.2 \mathrm{~cm}$ $\mathrm{TV} \times 1.7 \mathrm{~cm} \mathrm{SI}$ with increased mass effect on the optic chiasm. right upper quadrantanopsia, and a follow-up MRI at 2 years found no new regrowth of her craniopharyngioma.

\section{Discussion}

Although Cushing and Bailey first described postoperative aseptic meningitis in 1929, it remains a rare and enigmatic clinical entity. ${ }^{3}$ This case report highlights the difficulties in the diagnosis and treatment of patients with aseptic meningitis after surgical resection of craniopharyngiomas. The precise incidence of aseptic meningitis after craniopharyngioma removal remains undetermined in the literature. A retrospective review of 64 patients who had undergone endoscopic endonasal surgery for craniopharyngioma found that $7.8 \%$ were diagnosed with postoperative meningitis, but this study did not delineate between infectious and noninfectious etiologies. ${ }^{7}$ In a large series of 1,146 patients followed after removal of tumors at the cerebellopontine angle, the overall rate of meningitis was $4.54 \%$, and the rate of aseptic meningitis was $2.61 \%$, suggesting that aseptic meningitis represents a substantive portion of all postoperative cases. ${ }^{1}$

Aseptic meningitis is caused by various irritants that can trigger inflammatory responses at the meninges. Possible mechanisms include the direct intraoperative manipulation of vessels, the inflammatory effects of red blood cells, and the release of cystic fluids from intracranial masses. ${ }^{8}$ Craniopharyngiomas have specifically been implicated in several case reports to cause aseptic meningitis through spontaneous cyst rupture and release of cholesterol-filled contents. ${ }^{6,9,10}$ This resulting aseptic inflammation has also been linked to further complications, including thromboembolic events, cerebrovascular vasospasm, and even death. ${ }^{10-12}$

In this present report, the patient first suffered symptoms of cerebral vasospasm 18 days after her original transsphenoidal surgery. A small number of cases in the literature describe vasospasm as a complication of skull base tumor surgery. ${ }^{10,13,14}$ Various conditions can trigger vasospasm: direct mechanical damage to arterial walls, chemical substances released during tumor resection, surgical manipulation of the hypothalamus, and/or inflammation surrounding the breakdown of blood in the 
e154 Aseptic Meningitis with Craniopharyngioma Resection Chen et al.

Table 1 Clinical and laboratory findings associated with infectious versus aseptic meningitis ${ }^{1,19}$

\begin{tabular}{|l|l|l|}
\hline & Bacterial meningitis & Aseptic meningitis \\
\hline Symptoms & $\begin{array}{l}\text { Meningismus: nuchal rigidity, photophobia, } \\
\text { headache, mental status change }\end{array}$ & $\begin{array}{l}\text { Meningismus: nuchal rigidity, photophobia, } \\
\text { headache, mental status change }\end{array}$ \\
\hline Fever & $>102^{\circ} \mathrm{F}$ & $<102^{\circ} \mathrm{F}$ \\
\hline CSF rhinorrhea & Potentially present & Absent \\
\hline CSF culture/gram stain & Positive & Negative \\
\hline CSF lactate & $>35 \mathrm{mg} / \mathrm{dL}$ (sensitivity 0.93, specificity 0.99$)$ & $<35 \mathrm{mg} / \mathrm{dL}$ \\
\hline CSF WBC & $>6,000$ cells/ $\mu \mathrm{L}$ & $<2,000$ cells $/ \mu \mathrm{L}$ \\
\hline CSF glucose ${ }^{a}$ & $<20 \mathrm{mg} / \mathrm{dL}$ & Normal \\
\hline CSF protein & Not statistically different & Not statistically different \\
\hline
\end{tabular}

Abbreviations: CSF, cerebrospinal fluid; WBC, white blood cells.

${ }^{a}$ In nondiabetic patients.

basal cisterns. ${ }^{13}$ Craniopharyngioma fluid has also been shown to directly cause arterial spasm in animal experiments. ${ }^{15}$ Thus, the same chemical environment that can trigger aseptic meningitis may also lead to serious cerebrovascular complications, as experienced by the patient in this report. Consequently, aseptic meningitis and the development of associated complications should remain high on the differential in patients presenting with fever or meningismus after craniopharyngioma resection.

When clinically suspected, both the diagnosis and treatment of aseptic meningitis remains challenging. Diagnostically, there are few clear clinical findings that distinguish aseptic from bacterial meningitis as both can present with symptoms of headache, altered mental status, photophobia, and nuchal rigidity. Smaller studies have hypothesized that fevers over $102^{\circ} \mathrm{F}$, fevers lasting longer than 7 days, or a reported period of unconsciousness are more suggestive of bacterial meningitis. ${ }^{16}$ However, the largest study in the literature that followed 1,146 patients postoperatively was unable to find a statistically significant difference in fever on presentation. ${ }^{1}$ As aseptic meningitis remains largely a case reportable phenomenon and may be underreported given diagnostic challenges, the sample size available in the literature may remain insufficient to garner meaningful conclusions.

The laboratory workup of meningitis includes lumbar puncture with CSF analysis and culture, along with serum white blood cell count and brain MRI to rule out abscess in the postoperative setting. CSF culture growth of an infectious agent clearly differentiates an infectious etiology from aseptic meningitis. However, infectious meningitis may yield sterile cultures, particularly when empiric antibiotics are initiated before obtaining culture samples. ${ }^{17}$ Gram stain sensitivities for bacterial meningitis have been reported at 56 to $86 \% .^{18}$ Thus, our patient's first episode of meningitis was difficult to definitively diagnose as an aseptic phenomenon and so she was started on a long course of antibiotics. During each of her admissions, lumbar punctures showed only mild leukocytosis of less than 2,000 cells/ $\mu \mathrm{L}$, while studies suggest that patients with greater than 6,000 cells $/ \mu \mathrm{L}$ in the CSF are more likely to have bacterial meningitis. ${ }^{1}$ Additional studies have shown that CSF glucose is significantly lower in patients with bacte- rial meningitis, while CSF and serum white blood cells are significantly higher. ${ }^{1}$ In the literature, CSF lactate is a promising new biomarker in the early distinction of bacterial and aseptic meningitis, with one meta-analysis reporting a sensitivity and specificity of 93 and $96 \%$, respectively, for the diagnosis of bacterial meningitis. ${ }^{19}$ A clinical value of $35 \mathrm{mg} /$ $\mathrm{dL}$ was the recommended CSF lactate level cutoff. Taken together, patients with low-grade fevers, normal CSF glucose, low CSF lactate, and only mild CSF, and/or serum leukocytosis are likely to have aseptic meningitis. Clinical and laboratory characteristics associated with bacterial and aseptic meningitis are described in -Table 1. Collaboration with infectious disease specialists may be required to make the diagnosis of postoperative aseptic meningitis.

A high clinical suspicion for aseptic meningitis after craniopharyngioma resection is necessary to determine the optimal course of treatment. Patients with a high likelihood of aseptic meningitis may benefit from conservative management with steroids rather than prolonged courses of antibiotics or further surgical exploration. ${ }^{20}$ As the initial presentation may be difficult to distinguish from that of bacterial meningitis, the British Society of Antimicrobial Chemotherapy and various study authors have proposed treatment with empiric antibiotics for all patients presenting with meningitis after skull base surgery, but treatment should be discontinued when cultures return sterile in 48 or 72 hours. ${ }^{16,21}$ A cohort study of 75 patients with postoperative meningitis found that stopping antibiotics after 3 days was effective and safe for patients with negative CSF cultures. $^{2}$ At this time, regional preferences dictate the course and choice in antibiotics and close consultation with infectious disease specialists remains critical to providing optimal care. ${ }^{1,2}$ For each case it is prudent to balance the dangers of potential bacterial meningitis against the potential harms of long-term antibiotics and surgical re-exploration.

\section{Conclusion}

Aseptic meningitis must be considered in the febrile or meningitic patient after craniopharyngioma resection. Workup for an infectious etiology, including MRI and lumbar 
puncture, is imperative. CSF lactate may be useful in distinguishing between aseptic and bacterial meningitis, particularly in presentations of recurrent postoperative meningitis when cultures are sterile. Initial management with empiric, broad-spectrum antibiotics is prudent given the potentially devastating sequelae of infectious meningitis. However, in patients recovering from endoscopic resection of a craniopharyngioma without active rhinorrhea and supporting evidence for aseptic meningitis, a more conservative treatment course should be considered.

\section{Funding}

None.

\section{Conflict of Interest}

None.

\section{Acknowledgments}

None.

\section{References}

1 Sanchez GB, Kaylie DM, O'Malley MR, Labadie RF, Jackson CG, Haynes DS. Chemical meningitis following cerebellopontine angle tumor surgery. Otolaryngol Head Neck Surg 2008;138(3):368-373

2 Zarrouk V, Vassor I, Bert F, et al. Evaluation of the management of postoperative aseptic meningitis. Clin Infect Dis 2007;44(12): 1555-1559

3 Cushing H, Bailey P. Tumors arising from the blood vessels of the brain. Angiomatous malformations and hemangioblastomas 1928. Print. Thomas, Springfield

4 Finlayson AI, Penfield W. Acute postoperative aseptic leptomeningitis: Review of cases and discussion of pathogenesis. Arch Neurol Psychiatry 1941;46(2):250-276

5 Kaufman HH, Carmel PW. Aseptic meningitis and hydrocephalus after posterior fossa surgery. Acta Neurochir (Wien) 1978;44(3-4): 179-196
6 Satoh H, Uozumi T, Arita K, et al. Spontaneous rupture of craniopharyngioma cysts. A report of five cases and review of the literature. Surg Neurol 1993;40(5):414-419

7 Koutourousiou M, Gardner PA, Fernandez-Miranda JC. TylerKabara EC, Wang EW, Snyderman CH. Endoscopic endonasal surgery for craniopharyngiomas: surgical outcome in 64 patients. J Neurosurg 2013;119(5):1194-1207

8 Blomstedt GC. Post-operative aseptic meningitis. Acta Neurochir (Wien) 1987;89(3-4):112-116

9 Krueger DW, Larson EB. Recurrent fever of unknown origin, coma, and meningismus due to a leaking craniopharyngioma. Am J Med 1988;84(3 Pt 1):543-545

10 Shida N, Nakasato N, Mizoi K, Kanaki M, Yoshimoto T. Symptomatic vessel narrowing caused by spontaneous rupture of craniopharyngioma cyst-case report. Neurol Med Chir (Tokyo) 1998;38(10): 666-668

11 Fischer B, Palkovic S, Schildheuer D, Heindel W, Wassmann H. Inflammatory thromboembolic complication after craniopharyngioma surgery? Acta Neurochir (Wien) 2004;146(2):183-186

12 Hadden D, Allen I. Chemical meningitis due to rupture of a craniopharyngioma cyst. J R Soc Med 2004;97(12):585-586

13 Aoki N, Origitano TC, al-Mefty O. Vasospasm after resection of skull base tumors. Acta Neurochir (Wien) 1995;132(1-3):53-58

14 Alotaibi NM, Lanzino G. Cerebral vasospasm following tumor resection. J Neurointerv Surg 2013;5(5):413-418

15 Kamal R, Jindal A, Suri A, Mahapatra AK. Effect of craniopharyngioma fluid on femoral vessels of rat. Neurol Res 1999;21(8):796-798

16 Forgacs P, Geyer CA, Freidberg SR. Characterization of chemical meningitis after neurological surgery. Clin Infect Dis 2001;32(2):179-185

17 van de Beek D, Drake JM, Tunkel AR. Nosocomial bacterial meningitis. N Engl J Med 2010;362(2):146-154

18 Straus SE, Thorpe KE, Holroyd-Leduc J. How do I perform a lumbar puncture and analyze the results to diagnose bacterial meningitis? JAMA 2006;296(16):2012-2022

19 Sakushima K, Hayashino Y, Kawaguchi T, Jackson JL, Fukuhara S. Diagnostic accuracy of cerebrospinal fluid lactate for differentiating bacterial meningitis from aseptic meningitis: a meta-analysis. J Infect 2011;62(4):255-262

20 Carmel PW, Greif LK. The aseptic meningitis syndrome: a complication of posterior fossa surgery. Pediatr Neurosurg 1993;19(5):276-280

21 Infection in Neurosurgery Working Party of the British Society for Antimicrobial Chemotherapy. The management of neurosurgical patients with postoperative bacterial or aseptic meningitis or external ventricular drain-associated ventriculitis. $\mathrm{Br} \mathrm{J}$ Neurosurg 2000;14(1):7-12 